\title{
DINÂMICA E BALANÇO DO CARBONO DA VEGETAÇÃO PRIMÁRIA DA AMAZÔNIA CENTRAL
}

\author{
Niro Higuchi ${ }^{1}$ \\ Jeffrey Chambers ${ }^{2}$ \\ Joaquim dos Santos ${ }^{3}$ \\ Ralfh João Ribeiro ${ }^{4}$ \\ Alberto Carlos Martins Pinto ${ }^{5}$ \\ Roseana Pereira da Silva ${ }^{5}$ \\ Rosana de Miranda Rocha ${ }^{5}$ \\ Edgard Siza Tribuzy ${ }^{6}$
}

\begin{abstract}
RESUMO
As três parcelas permanentes usadas neste estudo são testemunhas (não perturbadas) de um experimento de manejo florestal do Instituto Nacional de Pesquisas da Amazônia, no município de Manaus (AM). Essas parcelas têm sido monitoradas desde 1980, mas para efeito deste estudo, foram consideradas 12 medições repetidas no período 19862000. Durante este período, o fenômeno El Niño (seca anormal na região) ocorreu em duas ocasiões, em 1992-93 e 1997-98, sendo que o último foi seguido do La Niña (chuva anormal na região), em 1999. Devido a esses fenômenos, as taxas de recrutamento e mortalidade foram iguais, $0,7 \%$, durante o período observado. No entanto, a acumulação (fixação na árvore) de carbono, foi de 16 toneladas métricas, dando um incremento periódico anual significativo ( $\mathrm{p}=$ $0,039)$, em torno de 1,2 t/ha/ano.

Palavras-chave: biomassa, parcelas permanentes, mudanças climáticas
\end{abstract}

\section{CARBON BALANCE AND DYNAMICS OF PRIMARY VEGETATION IN THE CENTRAL AMAZON}

\begin{abstract}
The three permanent forest inventory plots used for this study were control plots (not disturbed) from a forest management project of the National Institute of Amazon Research (INPA) in the Brazilian State of Amazonas. These plots have been monitored since 1980, although for this study the period from 1986-2000 was considered. During this period, the El Niño phenomenon, which causes increased drought in the region, occurred on two occasions (1992-93 and 1997-98), followed by La Niña which causes increased precipitation in the region (1999-2000). Despite of this change in climate, recruitment and mortality rates were equal throughout the period at $0.7 \% \mathrm{yr}-1$. During the same period, carbon accumulation in forest biomass was $16 \mathrm{Mg}$, resulting in a statistically significant $(\mathrm{p}=$ 0.039 ) increase of about $1.2 \mathrm{Mg}$ biomass ha-1 yr-1.
\end{abstract}

Keywords: biomass, repeated measurements, climate changes

\section{INTRODUÇÃO}

A obtenção de estimativas confiáveis de taxas de desmatamento e de estoques de carbono da vegetação é essencial para estimar a quantidade de carbono, emitida ou seqüestrada, no tempo e no espaço, principalmente na região amazônica. Sabendo a taxa de desmatamento, o passo seguinte é saber a quantidade de carbono da floresta que é convertida em outras formas

\footnotetext{
${ }^{1}$ Pesquisador, Dr., INPA, Coordenação de Pesquisas em Silvicultura Tropical, Manaus - AM, Brasil. niro@inpa.gov.br

${ }^{2}$ Pesquisador, Dr., Department of Ecology, Evolution and Marine Biology, University of California. Santa Barbara, CA 93106 USA.

${ }^{3}$ Pesquisador, Dr., Coordenação de Pesquisas em Silvicultura Tropical, Manaus - AM, Brasil. joca@inpa.gov.br.

${ }^{4}$ Pesquisador, MSc, Coordenação de Pesquisas em Silvicultura Tropical, Manaus - AM, Brasil. ralfh@inpa.gov.br.

${ }^{5}$ Bolsistas DCR-CNPq, MSc, Coordenação de Pesquisas em Silvicultura Tropical, Manaus - AM, Brasil

${ }^{6}$ Doutorando CENA-USP
}

Recebido para publicação: 15/04/2003

Aceito para publicação: 03/09/2004 
de uso do solo, para uma correta avaliação da participação dos projetos de desenvolvimento da região, no processo de mudanças climáticas globais (Fearnside et al. 1993; Foster Brown et al. 1995; Higuchi \& Carvalho Jr., 1995; Skole et al. 1994; Schroeder \& Winjum, 1995 e Fearnside, 1996), no âmbito da Convenção do Clima e do Protocolo de Quioto. Igualmente importante é o estudo da capacidade da floresta primária em manter o funcionamento natural das trocas gasosas entre a atmosfera e a biosfera.
As taxas de desmatamento na Amazônia eram, até os anos 90, apresentadas com muitas distorções e geravam polêmicas. Hoje, as estimativas fornecidas pelo INPE (2001) são mais consistentes. $\mathrm{O}$ total acumulado de área desmatada é de $569.269 \mathrm{~km}^{2}$ ou 56,9 milhões hectares, até 1999. As estatísticas de desmatamento anual são apresentadas na tabela 1 para cada estado da Amazônia, dando uma taxa anual média de desmatamento para toda a região de $17.065 \mathrm{~km}^{2}(\mathrm{~s}=4.882)$.

Tabela 1: Áreas anuais desmatadas $\left(\mathrm{km}^{2}\right)$ na Amazônia brasileira

Table 1: Deforested annual areas $\left(\mathrm{km}^{2}\right)$ in the Brazilian Amazony by state and by yer

\begin{tabular}{cccccccccccc}
\hline Estado & $78 / 87$ & $87-89$ & $89 / 90$ & $90 / 91$ & $91 / 92$ & $92 / 94$ & $94 / 95$ & $95 / 96$ & $96 / 97$ & $97 / 98$ & $98 / 99$ \\
\hline Acre & 620 & 540 & 550 & 380 & 400 & 482 & 1.208 & 433 & 358 & 536 & 441 \\
Amapá & 60 & 130 & 250 & 410 & 36 & 0 & 9 & 0 & 18 & 30 & 0 \\
Amazona & 1.510 & 1.180 & 520 & 980 & 799 & 370 & 2.114 & 1.023 & 589 & 670 & 720 \\
$\quad$ s & & & & & & & & & & & \\
Maranhã & 2.450 & 1.420 & 1.100 & 670 & 1.135 & 372 & 1.745 & 1.061 & 409 & 1.012 & 1.230 \\
$\quad$ o & & & & & & & & & & & \\
Mato & 5.140 & 5.960 & 4.020 & 2.840 & 4.674 & 6.220 & 10.391 & 6.543 & 5.271 & 6.466 & 6.963 \\
Grosso & & & & & & & & & & & \\
Pará & 6.990 & 5.750 & 4.890 & 3.780 & 3.787 & 4.284 & 7.845 & 6.135 & 4.139 & 5.829 & 5.111 \\
Rondônia & 2.340 & 1.430 & 1.670 & 1.110 & 2.665 & 2.595 & 4.730 & 2.432 & 1.986 & 2.041 & 2.368 \\
Roraima & 290 & 630 & 150 & 420 & 281 & 240 & 220 & 214 & 184 & 223 & 220 \\
Tocantin & 1.650 & 730 & 580 & 440 & 409 & 333 & 797 & 320 & 273 & 576 & 216 \\
s & & & & & & & & & & & \\
\hline TOTAL & 21.130 & 17.860 & 13.810 & 11.130 & 13.786 & 14.896 & 29.059 & 18.161 & 13.227 & 17.383 & 17.269 \\
\hline
\end{tabular}

Fonte: INPE (2001)

\section{Total acumulado até agosto/99 $=\mathbf{5 6 9 . 2 6 9}$ km2.}

A estimativa do estoque de carbono da vegetação pode ser obtida pelo produto da biomassa florestal e concentração de carbono. A concentração de carbono na vegetação obtida por Higuchi e Carvalho Jr. (1994), está em torno de $48 \%$, valor este que está dentro dos limites de concentrações em florestas tropicais, entre 46 e $52 \%$. Segundo Brown et al. (1989), as estimativas de biomassa, atualmente disponíveis na literatura, dos diversos tipos florestais da Amazônia, vêm de estudos que utilizam métodos diretos e indiretos.

O método direto consiste na derrubada e pesagem de todas as árvores que ocorrem em parcelas fixas, fornecendo estimativas que não são confiáveis porque se baseiam em poucas parcelas, pequenas e tendenciosamente escolhidas (Brown et al. 1989). No método indireto, as estimativas têm sido produzidas a partir de dados de inventários florestais, que foram executados com a finalidade de planejar a exploração e o manejo florestal, sendo o volume da madeira, a principal variável. Neste método, a biomassa é estimada a partir do volume da madeira, usando-se a densidade média da madeira e um fator de correção para árvores com $\mathrm{DAP}<25 \mathrm{~cm}$.

Esses dois métodos ainda geram muita polêmica e controvérsias e produzem estimativas desencontradas, mesmo quando se usa o mesmo banco de dados (Fearnside et al., 1993; Brown et al., 1989; Higuchi et al., 1994 e Foster Brown et al., 1995). A tabela 2 ilustra o que foi posto anteriormente. Esta tabela foi parcialmente reproduzida de Fearnside et al. (1993), considerando apenas a biomassa viva acima do nível do solo. São produzidas estimativas diferentes, com o passar do tempo, pelos mesmos autores e para o mesmo banco de dados (montado nos anos 70). Além disso, Foster Brown et al. (1995) criticam estes bancos de dados, afirmando que as alturas das árvores foram obtidas sem aparelhos de medição e que esses erros não-amostrais não são mencionados. 
Tabela 2: Algumas estimativas de biomassa seca para a floresta densa da Amazônia brasileira*

Table 2: $\quad$ Some dry biomass estimates for the Brazilian Amazony density forest

\begin{tabular}{|c|c|c|}
\hline Tipo de floresta & biomassa $(\mathrm{t})$ & Fonte \\
\hline Densa (RADAMBRASIL) & 268 & Brown \& Lugo $\left.\left(1992^{\mathrm{a}}\right)\right)-$ cf. fonte ${ }^{*}$ \\
\hline Densa (FAO) & 162 & Brown \& Lugo $\left(1992^{\mathrm{a}}\right)$ - cf. fonte ${ }^{*}$ \\
\hline Densa (RADAMBRASIL) & 289 & Brown \& Lugo (1992b) - cf. fonte ${ }^{*}$ \\
\hline Densa (FAO) & 227 & Brown \& Lugo (1992b) - cf. fonte ${ }^{*}$ \\
\hline Densa (presente) & 142 & Fearnside $\left(1992^{\mathrm{a}}\right)-$ cf. fonte $^{*}$ \\
\hline Densa (presente) & 320 & Fearnside (unpub. 1993) - cf. fonte ${ }^{*}$ \\
\hline
\end{tabular}

(*) Fonte: parcialmente reproduzida de Fearnside et al. (1993).

A maneira de estimar a biomassa florestal, a partir de dados de inventário, é utilizando a densidade média da madeira e um fator de correção para árvores com diâmetro à altura do peito (DAP) menor que $25 \mathrm{~cm}$. A densidade da madeira varia de espécie para espécie e, mesmo dentro da mesma espécie, há variações entre as diferentes partes da árvore (tronco, galhos grandes, galhos pequenos etc.) e, ainda dentro da mesma parte, há variações no sentido medula-casca. Em geral, a densidade média usada é a do DAP, fazendo-a menos consistente ainda.

É importante ressaltar também que, nenhum desses inventários utilizados por esses autores (Fearnside et al., 1993; Brown et al., 1989; Higuchi et al., 1994 e Foster Brown et al., 1995), foi executado com o objetivo de estimar a biomassa florestal. Os inventários dos anos $60 \mathrm{e}$ 70 se restringiam ao volume de madeira em tamanho comercial (normalmente considerando apenas árvores com DAP $>25 \mathrm{~cm}$ ), devido os mesmos serem exploratórios, servindo, no máximo, para estudo de viabilidade de projetos de desenvolvimento. Da mesma maneira, os volumes eram estimados por meio de um único fator de forma para todas as espécies da Amazônia. O fator de forma também varia de espécie para espécie e de sítio para sítio. Os inventários que não utilizam equações de volume, geralmente subestimam o volume da madeira.

Num ponto todos os grupos que trabalham com biomassa florestal concordam: estimar a biomassa acima do nível do solo de toda a Amazônia é simplesmente impossível por meio do método direto. Por esta razão, é preciso aperfeiçoar os métodos indiretos para se chegar a estimativas mais confiáveis. O ideal é ter um modelo estatístico para cada tipo florestal e para cada sítio, e para o caso de estudos de mudanças climáticas, é importante dar prioridade às áreas mais sujeitas às intervenções. Diante disso, é preciso ter uma boa idéia do mundo real, entendendo o relacionamento existente entre a biomassa florestal e outras variáveis da árvore, antes de se chegar ao mundo matemático. $\mathrm{Na}$ Amazônia Central, esta questão está parcialmente resolvida com o desenvolvimento e validação de equações alométricas para estimar a biomassa acima do nível do solo, de árvores em pé (Overman et al., 1994; Higuchi et al., 1994; Santos, 1996 e Higuchi et al., 1998).

O objetivo deste trabalho é analisar a dinâmica de uma floresta primária da Amazônia Central, no período de 1986 a 2000, quanto aos aspectos de recrutamento, mortalidade e crescimento do povoamento florestal. Usando as estimativas desses parâmetros, é possível entender o balanço do carbono da vegetação primária e saber se a floresta, nessas condições, está funcionando como fonte ou fixadora de carbono. Para atingir este objetivo, foram monitoradas três parcelas permanentes de um hectare cada, usadas como testemunhas de um experimento de manejo florestal. Nessas parcelas, todos os indivíduos arbóreos com diâmetro à altura do peito (DAP) maior ou igual a $10 \mathrm{~cm}$ são medidos anualmente. Foi usada a melhor equação alométrica para estimar a biomassa fresca acima do nível do solo e, depois disso, determinou-se o peso seco e o estoque de carbono individual.

\section{MATERIAL E MÉTODOS:}

\section{Pressupostos}

Os pressupostos deste estudo são: (i) $\mathrm{O}$ Protocolo de Quioto da Convenção sobre Mudança do Clima é uma realidade e, por conta disso, o mundo vai se mobilizar para atenuar o 
efeito estufa; (ii) No Brasil, a emissão de carbono via uso do solo amazônico é mais importante do que a queima de combustível fóssil; (iii) As taxas anuais de desmatamento na Amazônia são altas e estáveis; (iv) Mais importante do que a capacidade da região amazônica em seqüestrar carbono via reflorestamento ou regeneração natural, é o papel da floresta primária em manter as trocas gasosas entre a biosfera e atmosfera; (v) $\mathrm{O}$ mito "pulmão do mundo" atribuído à Amazônia há muito tempo foi derrubado por dados científicos, que provam que a floresta natural está em equilíbrio quanto à produção de Oxigênio (Salati e Vose, 1984 e TCA, 1993) e (vi) Estudo de Grace et al. (1995) indica que a floresta de Rondônia, não perturbada, funciona como seqüestradora de Carbono.

\section{Hipótese científica}

A floresta amazônica, em condições naturais, está em "steady state," ou seja, o balanço entre as taxas de mortalidade e de recrutamento mais o crescimento individual das árvores, determinam a velocidade com que a floresta vai da capacidade mínima à capacidade máxima de suporte do sítio.

\section{Caracterização da área}

$\mathrm{O}$ estudo está sendo realizado na área experimental de manejo florestal do INPA, na Estação ZF-2. O experimento cobre uma área de aproximadamente 2.000 ha, dentro da reserva florestal de 21.000 ha do INPA. A área localizase entre as coordenadas são: $2^{\circ}$ e $37^{\prime}$ a $2^{\circ}$ e $38^{\prime}$ de latitude Sul e $60^{\circ} 09^{\prime}$ a $60^{\circ}$ e $11^{\prime}$ de longitude Oeste de Grw., com base no mapa do Radambrasil (folha SA-20-Z-B), sittuando-se no distrito agropecuário da SUFRAMA, entre os km-21 e km-24 da vicinal ZF-2 (lado sul), perpendicular a BR-174 (na altura do km-50).

A cobertura florestal da EEST pode ser considerada como típica da floresta tropical úmida densa de terra-firme. Este tipo florestal é predominante na região amazônica, com aproximadamente 300 milhões de hectares (Braga, 1979). As famílias botânicas dominantes na área de estudo são: Sapotaceae, Lecythidaceae, Leguminosae e Burseraceae. As espécies que caracterizam esta área são Dinizia excelsa Ducke (Angelim-pedra), Eschweilera coriaceae (DC.) S.A. Mori (matá-matá), Protium apiculatum Swartz (breu-vermelho) e muitas outras espécies de Protium, Scleronema micranthum Ducke (cardeiro), Micrandropsis scleroxylon W. Rodr. (piãozinho), várias espécies de Pouteria, Ecclinusa, Micropholis, e Manilkara - abiuranas - várias espécies de Ocotea (louros) e algumas espécies de Parkia (faveiras).

Segundo Ranzani (1980), o clima é do tipo Am, classificação de Köppen, quente e úmido, com precipitação acima de $2.000 \mathrm{~mm}$ por ano, com período relativamente seco (junho a outubro). A temperatura média anual é de $27^{\circ} \mathrm{C}$ $\left(24\right.$ a $\left.31^{\circ} \mathrm{C}\right)$. Geologicamente predominam os arenitos cauliníticos, argilitos, grauvacas e brechas intraformacionais na formação Alter do Chão, do Cretáceo superior (Ranzani, 1980). A planície sedimentar Terciária na área é dissecada por sua rede de drenagem, resultando em platôs (de 500 a 1.000 metros de diâmetro), colinas de topos arredondados e vales amplos circundados por vertentes íngremes retas e convexas. A altitude relativa entre os vales e os platôs varia entre 50 a 100 metros. Segundo Ranzani (1980), há predominância de solos do tipo Latossolo amarelo distrófico de textura argilosa.

\section{Descrição do experimento}

$\mathrm{O}$ experimento de manejo florestal é composto de 3 blocos ou repetições de 24 hectares $(400 \times 600 \mathrm{~m})$ cada e 6 tratamentos de 4 hectares $(200 \times 200 \mathrm{~m})$ cada, totalizando 72 hectares, descrito por Higuchi et al. (1985). Os tratamentos são: testemunha, 4 diferentes intensidades de corte e anelamento. As parcelas permanentes de 1 hectare $(100 \times 100 \mathrm{~m})$ cada, estão instaladas no centro dos tratamentos, ou seja, cada tratamento tem um tampão de $100 \mathrm{~m}$, que anula os efeitos de borda apontados por Laurance et al. (2001). Este estudo usa apenas as três repetições da testemunha, parcelas não exploradas, que desde 1986 são monitoradas incluindo identificações de todas as árvores com $\mathrm{DAP} \geq 10 \mathrm{~cm}$, medições de DAP e observações sobre o recrutamento de novos indivíduos para classe de DAP mínimo e mortalidade. 


\section{Estimativas das variáveis utilizadas:}

i) Peso da massa fresca:

Foi estimado usando as equações alométricas desenvolvidas por Higuchi et al. (1998) separadamente, para indivíduos com DAP entre 10 e $20 \mathrm{~cm}$ e para indivíduos com DAP $\geq 20 \mathrm{~cm}$, da seguinte maneira:

$$
\begin{aligned}
& \ln \mathrm{PF}=-1,754+2,665 \text { ln } \mathrm{DAP} \\
& (10 \leq \mathrm{DAP}<20 \mathrm{~cm}) ; \mathrm{r}^{2}=0,92 \\
& \ln \mathrm{PF}=-0,151+2,17 \ln \mathrm{DAP}(\mathrm{DAP} \geq 20 \\
& \mathrm{cm}) ; \mathrm{r}^{2}=0,90 \\
& \ln =\text { logaritmo natural; } \\
& \mathrm{PF}=\text { peso seco da biomassa em } \mathrm{kg} \mathrm{e} \\
& \mathrm{DAP}=\text { diâmetro à altura do peito em } \mathrm{cm} ;
\end{aligned}
$$$$
\text { sendo: }
$$

ii) Peso seco:

Foi estimado pelo produto da massa fresca e a concentração de água em madeiras da região. Foi usada a concentração de água igual a $40 \%$, para toda a árvore, de acordo com as recomendações de Higuchi e Carvalho Jr. (1994). Neste mesmo estudo, as concentrações de água nas partes foram: 38,9\%, 39,4\%, $42,8 \%, 52,4 \%$ e $63,3 \%$, respectivamente para tronco, galhos grossos (diâmetro de base maior que $10 \mathrm{~cm}$ ), galhos finos, folhas e flores e/ou frutos. Por outro lado, a contribuição de cada parte da árvore ao seu peso total foi a seguinte: tronco $(65,6 \%)$, galhos grossos $(17,8 \%)$, galhos finos $(14,5 \%)$, folhas $(2 \%)$ e flores e/ou frutos $(0,01 \%)$.

\section{iii) Concentração de Carbono:}

Foi estimado pelo produto do peso da massa seca de cada indivíduo arbóreo e a concentração de Carbono igual a 48\% determinada por Higuchi e Carvalho Jr. (1994). Para a determinação da concentração de carbono foram coletadas amostras (discos), apenas do tronco, de 19 espécies mais comuns na área, tendo de 1 a 3 repetições, totalizando 44 árvores. De cada tronco, foram retirados dois discos, um da base e outro do topo, que foram secados em estufa até atingir o peso constante. $\mathrm{O}$ analisador elementar utilizado foi um Perkin Elmer modelo 2400. A média aritmética da concentração de $\mathrm{C}$ da base do tronco foi de $48,5 \%(\mathrm{~s}=1,6)$ e do topo de $48,2 \%(\mathrm{~s}=1,0)$, sendo o mínimo igual a $46 \%$ (base do breu 1) e o máximo igual a $53 \%$ (base da abiurana 1). A concentração de $\mathrm{C}$ nos galhos finos foi de $46,8 \%$.

iv) Estoque de carbono por hectare:

Os estoques foram estimados para as três repetições da parcela-testemunha, durante o período 1986-2000. A análise foi feita para o incremento acumulado durante o período.

v) Teste estatístico para o estoque de carbono:

Foi utilizada a análise de variância de simples entrada para medições repetidas usando as diferenças em acumulação de carbono na vegetação com o passar do tempo (de 1986 a 2000), de acordo com von Ende (1993).

vi) Taxas anuais de recrutamento e mortalidade:

Essas taxas foram determinadas entre duas ocasiões medidas. Recrutamento é o ingresso de um novo indivíduo à primeira classe de DAP $(D A P \geq 10 \mathrm{~cm})$ e mortalidade é a morte de um indivíduo ocorrida entre duas ocasiões.

\section{DISCUSSÃO DOS RESULTADOS}

O balanço de carbono da vegetação primária depende das taxas de mortalidade e recrutamento bem como do crescimento interno (mudanças de classes de diâmetro, individual). Em geral, o diâmetro das árvores mortas é sempre maior do que o das recrutas e, por essa razão, quando as taxas são iguais, o crescimento interno precisa compensar as perdas causadas pela mortalidade para que o ecossistema tenha um saldo positivo para qualificar-se como fixador de carbono e não como fonte.

Durante o período 1986-2000, as taxas de mortalidade e recrutamento oscilaram muito, sem uma definição clara de um padrão comportamental (figura 1). As médias das três repetições (blocos) foram $4,7 \%$ e $5,1 \%$, respectivamente, para mortas e recrutas; do ponto de vista relativo, as taxas anuais são iguais, ou seja, $0,7 \%$, tanto para a mortalidade como para o recrutamento. Em área contígua à área de estudo, usando bandas dendrométricas, Silva et al. (2001) concluíram que o incremento anual médio individual em diâmetro, considerando 272 diferentes árvores, foi de 1,64 mm, dando um crescimento de 1,64 $\mathrm{cm}$ em 10 anos. Neste caso, este crescimento 
interno compensou as perdas de biomassa viva

sofreram mortalidade. causadas pelo maior tamanho das árvores que

Figura 1: Taxas (\%) anuais de mortalidade e recrutamento de uma floresta não perturbada da Amazônia Central Figure 1: Annual rates of mortality and recruitment of an undistrebed Central Amazon forest

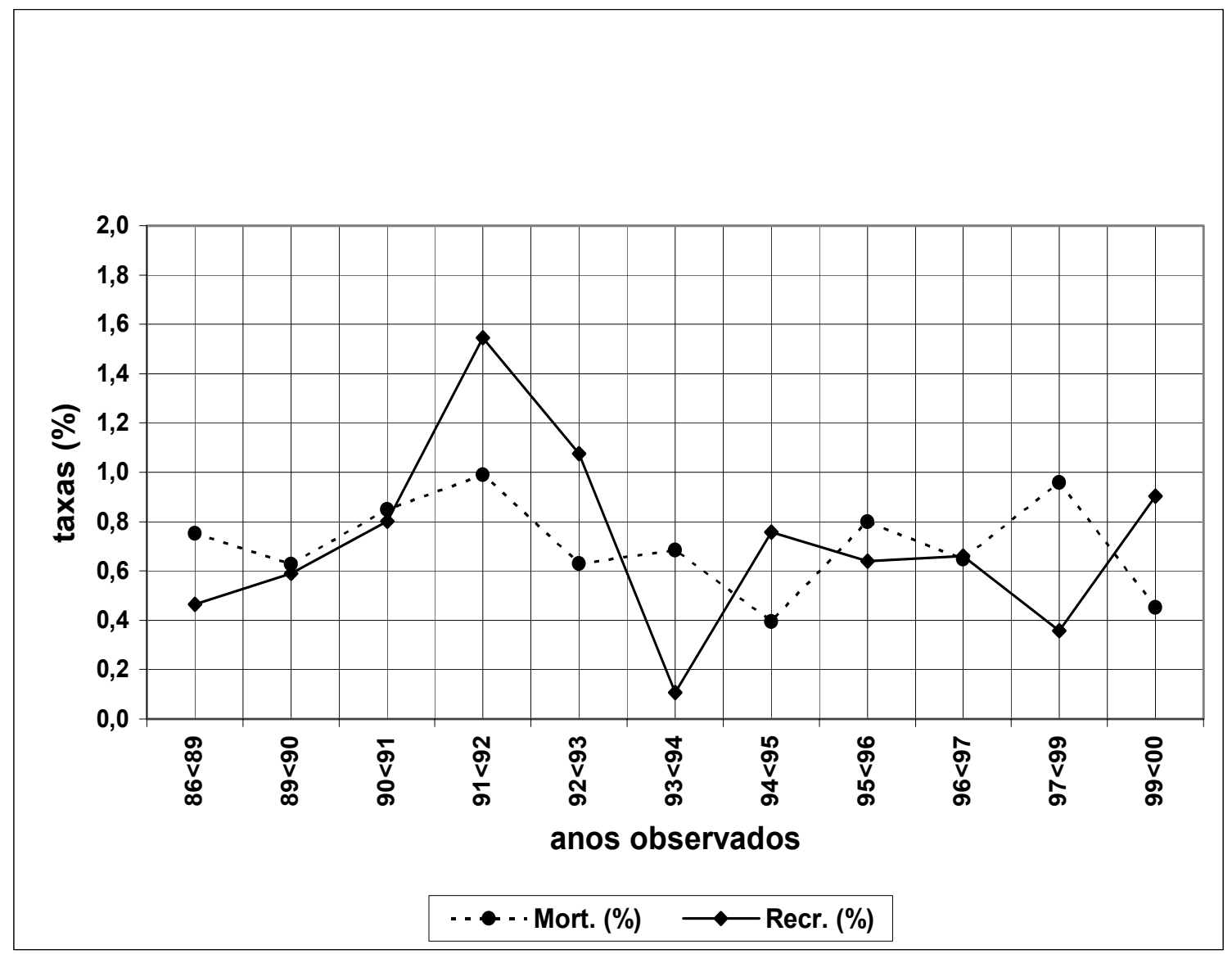

O fenômeno El Niño (muita seca na região de Manaus) ocorreu em 1992-93 e em 1997-98, sendo que o último foi seguido pelo La Niña (muita chuva na região), em 1999. Olhando a figura 1, em 1992-93, o El Niño não causou o aumento da mortalidade, apesar do stress hídrico; no entanto, entre os anos 1997 e 1999 (durante a ocorrência dos dois fenômenos), a mortalidade foi maior do que o recrutamento (tabela 3). Num primeiro momento, tem-se a impressão que o stress hídrico (causando morte da árvore em pé) provoca menos danos do que as chuvas torrenciais (normalmente acompanhadas de ventos fortes). Do ponto de vista estatístico, a correlação entre precipitação e mortalidade é negativa $(\mathrm{r}=-0,248)$, porém não significante $(\mathrm{p}=0,462)$. 
Tabela 3: Taxas anuais de mortalidade (M) e recrutamento (R) da floresta não perturbada da região de Manaus (AM)

Table 3: $\quad$ Rates of annual mortality $(M)$ and recruitment $(R)$ of the undisturbed forest of the Manaus region

\begin{tabular}{cccccc}
\hline Anos & $\mathrm{M}(\%)$ & $\mathrm{R}(\%)$ & mortas & recrutas & Total \\
\hline $86<89$ & 0,8 & 0,5 & 4,7 & 8,6 & 625 \\
$89<90$ & 0,6 & 0,6 & 3,9 & 3,8 & 637 \\
$90<91$ & 0,8 & 0,8 & 5,3 & 5,0 & 636 \\
$91<92$ & 1,0 & 1,5 & 6,3 & 9,7 & 636 \\
$92<93$ & 0,6 & 1,1 & 4,0 & 6,7 & 639 \\
$93<94$ & 0,7 & 0,1 & 4,3 & 0,7 & 642 \\
$94<95$ & 0,4 & 0,8 & 4,0 & 5,0 & 638 \\
$95<96$ & 0,8 & 0,6 & 6,0 & 4,3 & 639 \\
$96<97$ & 0,6 & 0,7 & 4,0 & 4,3 & 637 \\
$97<99$ & 1,0 & 0,4 & 6,2 & 2,2 & 637 \\
$99<00$ & 0,5 & 0,9 & 3,0 & 5,7 & 629 \\
\hline Média & 0,7 & 0,7 & 4,7 & 5,1 & 636 \\
\hline
\end{tabular}

Combinando a tabela 4 com a figura 2 , é possível perceber que a parcela-testemunha (floresta primária) acumulou $16 \mathrm{t}$ de carbono nas árvores durante o período observado, dando um incremento anual de 1,2 t/ha/ano. Este resultado está de acordo com a média de acumulação de carbono na Amazônia, igual a 0,97 t/ha/ano, estimada com base em 97 parcelas permanentes (Philips et al., 1998); da mesma forma, com o resultado de Grace et al. (1995), usando o método "eddy flux covariance", que foi de 1,9 t/ha/ano (acima e abaixo do nível do solo - todo o sistema). De um lado, esses resultados colocam um grande ponto de interrogação na hipótese do equilíbrio de todo o sistema amazônico (Salati e Vose, 1984), quanto ao balanço do carbono; de outro lado, essa acumulação pode explicar em parte, para onde está indo o "carbono perdido" estimado em 1,6 bilhão t/ano, segundo Houghton (1994).

Tabela 4: Dinâmica do carbono da vegetação (média anual) em t/ha/ano da floresta não perturbada da região de Manaus (AM)

Table 4: Vegetation carbon dynamic (annual average) in t/ha/ano of the undisturbed forest in the Manaus region

\begin{tabular}{ccccc}
\hline Anos & Bloco 1 & Bloco 2 & Bloco 3 & Média \\
\hline 1986 & 155 & 162 & 150 & 156 \\
1989 & 160 & 164 & 149 & 158 \\
1990 & 161 & 168 & 150 & 159 \\
1991 & 163 & 170 & 148 & 160 \\
1992 & 166 & 172 & 150 & 163 \\
1993 & 167 & 173 & 152 & 164 \\
1994 & 170 & 176 & 152 & 166 \\
1995 & 173 & 179 & 152 & 168 \\
1996 & 173 & 178 & 153 & 168 \\
1997 & 174 & 180 & 154 & 169 \\
1999 & 176 & 179 & 158 & 171 \\
2000 & 176 & 181 & 160 & 172 \\
\hline IPA & 1,50 & 1,37 & 0,72 & 1,20 \\
\hline
\end{tabular}


Figura 2: Dinâmica do carbono (média anual) de árvores com DAP $\geq 10 \mathrm{~cm}$ da floresta tropical úmida de terrafirme da região de Manaus (AM), de 1986 a 2000

Figure 2: Carbon dynamic (annual average) of trees with $D B H \geq 10 \mathrm{~cm}$ of the tropical rain forest of up land of the Manaus region from 1986 to 2000

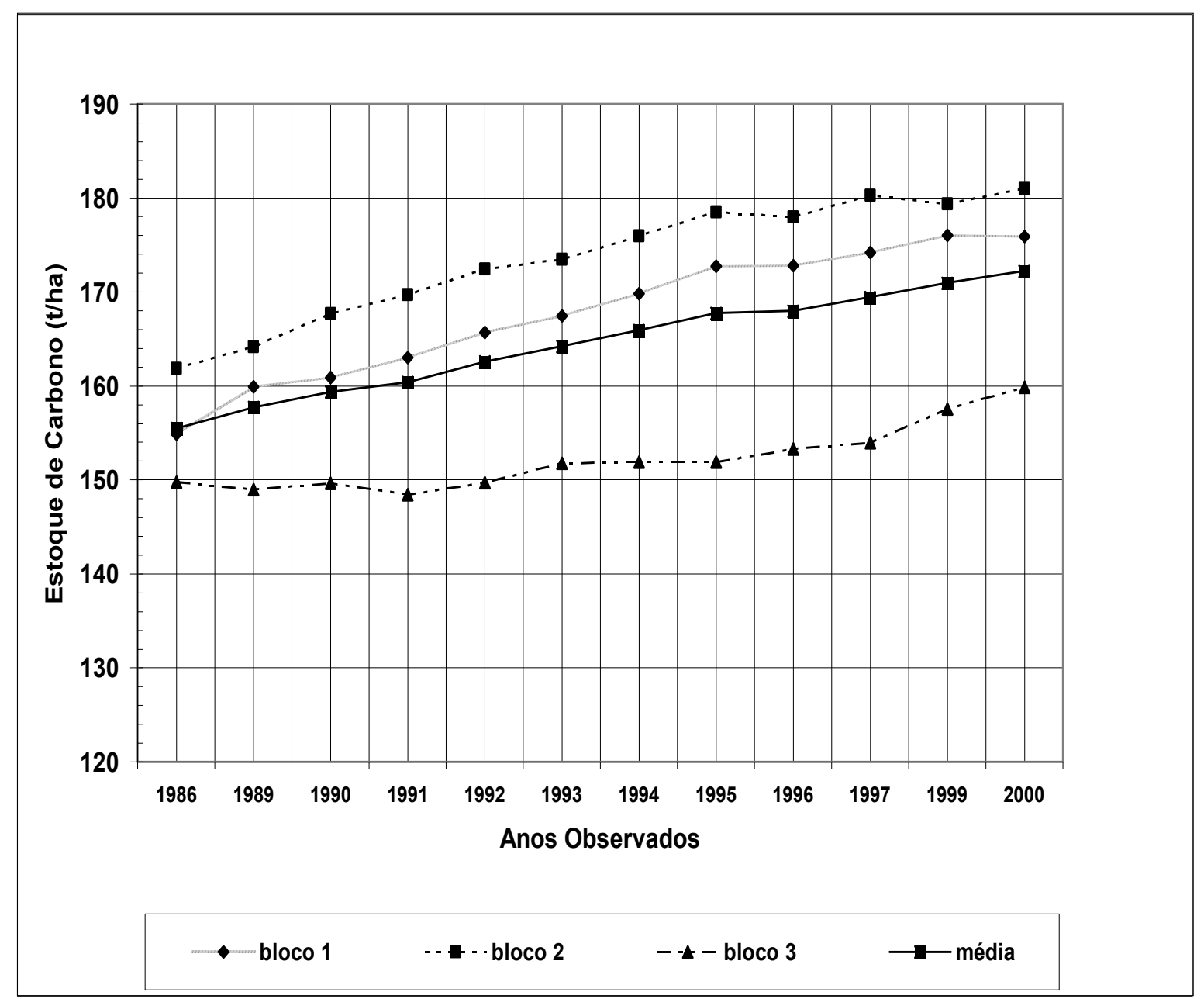

A tabela 5 apresenta a análise de variância de medições repetidas (12 medições em 14 anos), com base na acumulação anual de carbono. Como são medições repetidas, o teste-F é descartado e a inferência é feita com base nos fatores Greenhouse-Geisser (G-G) ou Huynh-Feldt (H-F), optando sempre pelo menor. Neste caso, o G-G é menor que H-F, sendo, por esta razão, mais conservador e mais seguro para inferir sem a influência do tempo e de medições sobre as mesmas parcelas permanentes. Dessa forma, podemos concluir que a acumulação anual é significante ao nível de $5 \%(p=0,039)$ e rejeitar a hipótese de que a floresta amazônica está em "steady state", pelo menos, para o período observado. 
Tabela 5: Análise de Variância de medições repetidas

Table 5: Analyse of variance of repeated measurements

\begin{tabular}{lccccccc}
\hline Fontes de Variação & GL & SQ & MQ & F & P & G-G & H-F \\
\hline Tempo & 10 & 710,5 & 71,0 & 17,9 & 0,000 & 0,039 & 0,010 \\
Resíduo & 20 & 79,5 & 4,0 & & & & \\
& & & & & & & \\
Greenhouse-Geisser Epsilon $=$ & 0,1162 & & & & & & \\
Huynh-Feldt Epsilon = & 0,1776 & & & & & & \\
\hline
\end{tabular}

\section{CONCLUSÃO}

As taxas anuais de mortalidade e recrutamento, durante o período 1986-2000, foram iguais, em torno de $0,7 \%$. No entanto, as acumulações de carbono, de ano para ano, foram significativas $(p=0,039)$, em torno de 1,2 t/ha/ano. $\mathrm{O}$ diâmetro médio das árvores mortas foi de $25 \mathrm{~cm}$, enquanto que o das recrutas foi de $12 \mathrm{~cm}$. Dessa forma, a acumulação só pode ser explicada pelo crescimento individual das árvores armazenadas dentro do sistema, compensando as perdas causadas pela mortalidade.

Considerando uma área de 100 milhões hectares na Amazônia Central, fisionomicamente similares à área de estudo, apenas como exercício de simulação linear, pode-se especular que, durante os últimos 14 anos, a floresta primária tem "seqüestrado" da atmosfera, 120 milhões toneladas de carbono por ano, que corresponde à metade do carbono emitido via desmatamento da Amazônia e quase o dobro da emissão brasileira via queima de combustível fóssil. Isso, no entanto, não ressuscita o mito "pulmão do mundo" por uma simples questão de escala, ou seja, enquanto o oxigênio participa com $21 \%$ da atmosfera, o carbono é um gás traço com uma contribuição de apenas $0,04 \%$.

Os resultados deste estudo são compatíveis com outros obtidos em outras regiões tropicais, sejam com o uso de parcelas fixas permanentes ou com o método "eddy flux covariance". Dentre as várias possíveis explicações para este comportamento, destacamse duas: (i) o equilíbrio das trocas gasosas entre a biosfera e atmosfera não é estático, mas sim dinâmico, ou seja, as florestas tropicais estão passando por uma fase de crescimento e se manterá dessa forma até atingir a capacidade máxima de suporte do sítio e (ii) a fertilização via poluição atmosférica. É possível que a poluição cause mudanças, principalmente, na velocidade que o ecossistema vai da capacidade mínima à máxima, assim como é possível que a poluição possa provocar, numa escala geológica, mudanças nos limites de capacidade de suporte dos sítios florestais. De qualquer modo, o crescimento observado no período de 14 anos não é linear, ou seja, não há qualquer chance da biomassa da floresta amazônica dobrar a cada 150 anos.

\section{AGRADECIMENTOS}

Ao CNPq pelas bolsas PQ (Niro Higuchi) e DCR (Roseana P. da Silva e Rosana M. Rocha); ao MCT pela bolsa PCI-DTI (Alberto C.M. Pinto) e aos projetos Jacaranda (Convênio INPA e JICA), Piculus (PPD/MCTFINEP/CCE) e LBA-Ecologia (MCT e NASA) pelo apoio logístico.

\section{REFERÊNCIAS}

Braga, P. I. S. Subdivisão fitogeográfica, tipos de vegetação, conservação e inventário florístico da floresta amazônica. Acta Amazonica, 9(4):53-80, 1979.

Brown, S.; Gillespie, A.. J. R. e Lugo, A. E. Biomass estimation methods for tropical forest with applications to forest inventory data. Forest Science, 35(4):881-902, 1989

Fearnside, P. M.; Leal Filho, N. e Fernandes, F. M. Rainforest burning and the global carbon budget: biomass, combustion efficiency, and charcoal formation in the Brazilian Amazon. J. of Geophysical Research, 98(D9):16733$16743,1993$.

Fearnside, P. M. Amazonian deforestation and global warming: carbon stocks in vegetation 
replacing Brazil's Amazon forest. Forest Ecology and Management, 80:21-34, 1996.

Foster Brown, I.; Martinelli, L. A.; Wayt Thomas, W.; Moreira, M. Z.; Cid Ferreira, C.A.; e Victória, R. Uncertainty in the biomass of Amazonian forests: an example from Rondônia. Forest Ecology and Management, 75:175-189, 1995.

Grace, J.; Lloyd, J.; McIntyre, J.; Miranda, A. C.; Meir, P.; Miranda, H. S.; Nobre, C.; Monc, J.; Massheder, J.; Malhi, Y.; Wright, I. e Gash., J. Carbon dioxide uptake by an undisturbed tropical rain forest in southwest Amazonia, 1992 to 1993. Science (270):778-780, 1995.

Higuchi. N., Jardim, F. C. S.; Santos J. dos e Barbosa, A. P. Bacia 3 - inventário florestal comercial. Acta Amazonica 15(3-4):327-369, 1985.

Higuchi, N.; Santos, J. M. dos, Imanaga, M. e Yoshida, S. Aboveground biomass estimate for Amazonian dense tropical moist forests. Memoirs of the Faculty of Agriculture, Kagoshima, 30(39):43-54, 1994.

Higuchi, N. e Carvalho Jr., J. A. Biomassa florestal e conteúdo de carbono de espécies arbóreas da Amazônia. Em: Emissão x Seqüestro de $\mathrm{CO}_{2}$ - Uma Nova Oportunidade de Negócios para o Brasil. CVRD. pp. 125-153, 1995.

Higuchi, N.; Santos, J. dos; Ribeiro, R. J.; Minette L. e Biot, Y. Biomassa da parte aérea da vegetação da floresta tropical úmida de terra-firme da Amazônia brasileira. Acta Amazonica, 28(2):153-166, 1998.

Houghton, R. A. As florestas e o ciclo de carbono global: armazenamento e emissões atuais. Em: Emissão x Seqüestro de $\mathrm{CO}_{2}$ Uma Nova Oportunidade de Negócios para o Brasil. CVRD. pp. 41-76. 1994.

INPE - Instituto Nacional de Pesquisas Espaciais. Desflorestamento na Amazônia. Homepage: www.inpe.br, 2001.

Laurance, W. F.; Lovejoy; T. E.; Vasconcelos; H. L.; Bruna; E. M.; Didham, F. K.; Stouffer, P. C.; Gascon, C.; Bierregaard Jr., R. O.; Laurance, S.G. e Sampaio, E. Ecosystem decay of Amazonian forest fragments: a 22-year investigation. Conservation Biology 16(3):605-618, 2001.

Overman, J. P. M.; Witte, H. J. L. e Saldarriaga, J.G. Evaluation of regression models for above-ground biomass determination in Amazonian rainforest. Forest Ecology and Management, 10:207-218, 1994.

Phillips, O. L., Malhi, Y.; Higuchi, N.; Laurance, W. F.; Núñez, P.V.; Vásquez, R. M.; Laurance, S. G.; Ferreira, L. V.; Stern, V.; Brown, S. e Grace, J. Changes in the carbon balance of tropical forests: evidence from long-term plots. Science, 282(5388):439-442, 1998.

Ranzani, G. Identificação e caracterização de alguns solos da Estação Experimental de Silvicultura Tropical. Acta Amazonica, 10(1):7-41, 1980.

Salati, E. e Vose, P. B. Amazon Basin: a system in equilibrium. Science 225(4658): 129-138, 1984.

Schroeder, P. E. e Winjum, J. K.. Assessing Brazil's carbon budget: II. Biotic fluxes and net carbon balance. Forest Ecology and Management, 75:87-99, 1995.

Santos, J. dos. Análise de modelos de regressão para estimar a fitomassa da floresta tropical úmida de terra-firme da Amazônia brasileira. Tese de Doutorado, Universidade Federal de Viçosa, 121 p. 1996.

Silva, R. P., Santos, J. dos; Tribuzi, E. S.; Chambers, J. Q.; Nakamura, S. e Higuchi, N. Diameter increment and growth patterns for individual trees growing in Central Amazon, Brazil. Forest Ecology and Management, 5725:1-7, 2001.

Skole, D. L.,. Chomentowski, W. H; Salas, W. A. e Nobre, A. D. Physical and human dimensions of deforestation in Amazônia. BioScience, 44(5):314-321, 1994.

Tratado de Cooperação Amazônica (TCA). Amazonia Without Myths. BID/TCA/ UNDP. 99 p. 1993.

Von Ende, C. N. Repeated-measures analysis: growth and other time-dependent measures. In: Schneider, S.M. e J. Gurevitch (eds.), Design and Analysis of Ecological Experiments. Chapman \& Hall, Londres, pp. 113-137, 1993. 